\title{
Coexpression Analysis Identified PcMYB25 as a Patchoulol Synthase Gene Activator to Enhance Patchouli Alcohol Biosynthesis in Pogostemon Cablin
}

\section{Xuanxuan Zhou}

Guangzhou University of Traditional Chinese Medicine: Guangzhou University of Chinese Medicine Xilin Wang

Guangzhou University of Traditional Chinese Medicine: Guangzhou University of Chinese Medicine Huiling Huang

Guangzhou University of Traditional Chinese Medicine: Guangzhou University of Chinese Medicine Daidi Wu

Guangzhou University of Traditional Chinese Medicine: Guangzhou University of Chinese Medicine Xiaobing Wang

Guangzhou University of Traditional Chinese Medicine: Guangzhou University of Chinese Medicine Baiyang Zhong

Guangzhou University of Traditional Chinese Medicine: Guangzhou University of Chinese Medicine Junren Li

Guangzhou University of Traditional Chinese Medicine: Guangzhou University of Chinese Medicine

\section{Xiuzhen Chen}

Guangzhou University of Traditional Chinese Medicine: Guangzhou University of Chinese Medicine

\section{Ruoting Zhan}

Guangzhou University of Traditional Chinese Medicine: Guangzhou University of Chinese Medicine Likai Chen ( $\square$ chenlk@gzucm.edu.cn )

Guangzhou University of Chinese Medicine https://orcid.org/0000-0001-7273-575X

\section{Original Article}

Keywords: WGCNA, patchouli alcohol, transcription factor, MYB

Posted Date: December 8th, 2021

DOI: https://doi.org/10.21203/rs.3.rs-207385/v2

License: (9) This work is licensed under a Creative Commons Attribution 4.0 International License. Read Full License 



\section{Coexpression analysis identified PcMYB25 as a patchoulol synthase}

\section{2 gene activator to enhance patchouli alcohol biosynthesis in}

\section{$3 \quad$ Pogostemon cablin}

4 Xuanxuan Zhou ${ }^{1}$, Xilin Wang ${ }^{1}$, Huiling Huang ${ }^{1}$, Daidi $\mathrm{Wu}^{1}$, Xiaobing Wang ${ }^{1}$, Baiyang Zhong ${ }^{1}$, 5 Junren $\mathrm{Li}^{1}$, Xiuzhen $\mathrm{Chen}^{1}$, Ruoting Zhan ${ }^{1,2,},{ }^{*}$, Likai Chen ${ }^{1,2,}{ }^{*}$

$6{ }^{1}$ Research Center of Chinese Herbal Resource Science and Engineering, Guangzhou University of Chinese Medicine;

Key Laboratory of Chinese Medicinal Resource from Lingnan (Guangzhou University of Chinese Medicine),

${ }^{2}$ Ministry of Education, Maoming Branch, Guangdong Laboratory for Lingnan Modern Agriculture, Guangdong,

China

\section{Authors' email:}

Xuanxuan zhou, 15521166528@163.com

Xilin Wang, 524996384@qq.com

Huiling Huang, hhl1107456909@126.com

Daidi Wu,w13726752703@163.com

19 Xiaobing Wang, 13570332896@163.com

Baiyang Zhong, 1642774256@qq.com

Xiuzhen Chen, xiuzhenchan@163.com 


\section{Abstract}

\section{Background}

Patchouli alcohol is an effective component of the medicinal plant patchouli. Similar to other secondary metabolites, its synthesis is likely also regulated by transcription factors. Although the biosynthetic pathway of patchouli alcohol has been characterized, the regulatory mechanism of patchouli alcohol biosynthesis has not been fully revealed.

\section{Results}

This study combined the transcriptome data of patchouli leaves treated with different hormones and WGCNA to establish a coexpression network. The modules correlated to patchouli alcohol content were identified, and PcMYB25 played a crucial role in regulating patchouli alcohol biosynthesis. The overexpression of PcMYB25 can promote the expression of patchouli alcohol synthase (PTS), thereby increasing the content of patchouli alcohol.

\section{Conclusions}

This is the first report that MYB25 regulates the secondary metabolism of patchouli. These experimental results lay the foundation for further analysis of the regulatory mechanism of patchouli alcohol synthesis.

\section{Key words:}

WGCNA, patchouli alcohol, transcription factor, MYB

\section{Background}

Pogostemon cablin (Blanco) Benth. is a kind of herbaceous plant that belongs to Labiatae and is distributed in Southern and Southeast Asia (Swamy and Sinniah 2016). The oil extracted from patchouli has been widely used for medical treatment, fragrances, and cosmetics. Patchouli oil is a special volatile oil, which contains more than 20 kinds of sesquiterpenes (Deguerry et al. 2006). Patchouli alcohol is the main component in the volatile oil of patchouli (Yan et al. 2021). It has been reported that the content of patchouli alcohol (PA) accounts for 
$55.7 \%$ of the oil extracted from the aerial parts of the plant (Lee et al. 2020). Patchouli alcohol is synthesized throughout the entire plant, and patchouli alcohol is mainly synthesized via the MVA pathway (Bouvier et al. 2005; Liao et al. 2016). The process of patchouli alcohol biosynthesis in patchouli includes three steps; first, isopentenyl pyrophosphate (IPP) and dimethylallylpyrophosphate (DMAPP) are mainly synthesized through the mevalonate aciddependent (MVA) pathway. The second step involves the synthesis of farnesyl diphosphate (FPP), which is catalyzed by farnesyl pyrophosphate synthase (FPPS). In the third step, PTS functions as an all-purpose sesquiterpene synthase and produces at least 13 sesquiterpenes (Deguerry et al. 2006).

In clinical medicine, patchouli alcohol has analgesic, anti-inflammatory, antidepressant, and anticancer effects (Lee et al. 2020). It is reported that patchouli alcohol can also be used as a food additive to prevent metabolic diseases, such as atherosclerosis. Because of patchouli alcohol's great application and commercial value, increasing the yield of patchouli alcohol by genetic engineering has become a hot research area. Mitsui et al. expressed the fusion proteins of FPPS and PTS in yeast, ultimately yielding $8.42 \mathrm{mg} / \mathrm{L} / \mathrm{d}$ (Mitsui et al. 2020). However, the biosynthesis of terpenoids is a complicated process and is affected by many rate-limiting enzymes. When only a few genes are modified, the anticipated effects are not always achieved. Additionally, transcription factors can simultaneously regulate multiple genes involved in terpenoid metabolism to achieve the accumulation of metabolites. For example, ORCA3 is a jasmonate-responsive transcription factor from Catharanthus roseus; the overexpression of ORCA3 upregulates the expression levels of the key enzyme genes AS, TDC, DXS, CPR, G10H, SLS, STR, SGD, and D4H in the biosynthetic pathway of terpene indole alkaloids, resulting in an increase of the production of TIA (Sun et al. 2020). The AP2/ERF family transcription factor AaORA in Artemisia annua is a positive regulator of genes such as $A a A D S$, $A a C Y P 71 A V 1, A a D B R 2$, and AaERF1. It can positively regulate the expression levels of these genes to increase the content of artemisinin. After AaORA overexpression, the content of artemisinin and dihydroartemisinic acid increased 40\% 53\% and 22\% 35\%, respectively (Lu et al. 2013; Shen et al. 2016).

In nature, the growth and development of plants are affected by many factors, such as temperature, humidity, and salinity. When sensing environmental changes, plants respond 
through hormone changes (Buchner et al. 2017; Jan et al. 2019; Verma et al. 2016). Altering concentration for various hormones could activate or inhibit activities of specific transcription factors, which in turn affects the synthesis and accumulation of secondary metabolites. Patchouli alcohol is a natural sesquiterpene; as a secondary metabolite, its synthesis is also affected by hormones and other signaling molecules. Wang et al. overexpressed PatJaz6 in patchouli, which led to the downregulation of PTS and a reduction in the content of patchouli alcohol in its leaves, indicating that PatJaz6 is a negative regulator of patchouli synthesis (Wang et al. 2019). Chen et al. showed through dual-luciferase experiments that the PatSWC4 transcription factor can bind to the promoter of PatPTS to increase its transcription activity. Yeast two-hybrid (Y2H) results indicated that PatSWC4 and PatJAZ4 proteins can interact, and suggested that PatSWC4 plays a part in the JA response network (Chen et al. 2020).

A good deal of research has been applied to reveal the regulatory network of patchouli alcohol biosynthesis for increasing its production. The rapid development of transcriptome sequencing technology and the continuous reduction in costs have led to a large amount of data support for seeking the functional genes. However, traditional comparative analysis is costly, and the data usually cannot be fully used. Weighted gene co-expression correlation network analysis (WGCNA) is a systematic biological analysis method that can correlate phenotypic trait data with specific genes. Currently, WGCNA plays an important role in the analysis of plant secondary metabolism. Lu et al. used WGCNA in combination with correlation analyses to screen out six transcription factors in chrysanthemum. It is speculated that these six transcription factors may affect the synthesis and accumulation of carotenoids in chrysanthemum by regulating genes involved in metabolism and plastid development (Lu et al. 2019). El-Sharkawy constructed a co-expression network for the differentially expressed genes in two kinds of apples and found 34 genes positively related to anthocyanin metabolism, and 12 of these genes in the anthocyanin metabolism pathway. It can be seen that WGCNA can be used to search for many functionally important genes (El-Sharkawy et al. 2015). However, there are few reports try to identify transcription factors related to patchouli alcohol metabolism by

\section{WGCNA.}

In this study, based on transcriptome sequencing data, the patchouli alcohol content is used as phenotypic data to link with gene modules according to WGCNA. A R2R3 MYB 
transcription factor gene, MYB25, was identified from these modules. Transient overexpression analysis confirmed that MYB25 is a positive regulator in the accumulation of patchouli alcohol. This study was the first that identified a transcription factor that positively regulates patchouli alcohol synthesis, laying the foundation for further research on the patchouli alcohol synthesis regulatory network in the future.

\section{Methods}

\section{Plant materials and treatment}

Pogostemon cablin (Blanco) Benth. was collected from Yangjiang, Guangdong, China (21.87 $\mathrm{N}, 111.98^{\circ} \mathrm{E}$ ), and grew in a growth chamber in the Research Center of Chinese Herbal Resource Science and Engineering, Guangzhou University of Chinese Medicine in Guangzhou University of Chinese Medicine $\left(23.03^{\circ} \mathrm{N}, 113.23^{\circ}\right.$ E). The temperature and humidity of the growth chamber was set to $26^{\circ} \mathrm{C} / 75 \%$ during the day and $21^{\circ} \mathrm{C} / 65 \%$ at night. Light/dark period was set to $16 / 8 \mathrm{~h}$

Six-leaf stage patchouli plants were used for hormone treatment. Patchouli plants with similar growth state were divided randomly into control ( $0.5 \%$ ethanol) and five phytohormonetreatment groups (MeJA, SA, ETH, ABA, and MeJA/ETH). Control ethanol and various hormones (dissolved in $0.5 \%$ ethanol) were applied on patchouli leaves by spraying. After spraying, the different treatment groups were enclosed by see-through plastic containers to prevent hormones from dissipating. Eight hours after spraying, leaves collected from each treatment group were frozen immediately in liquid nitrogen, and store at $-80^{\circ} \mathrm{C}$, used for RNASeq and extraction of patchouli alcohol. Concentrations used for various hormones are $300 \mathrm{M}$ (MeJA, SA, and ABA) or 500 M (ETH).

\section{Identification of DEGs and the co-expression network}

The transcriptome sequencing, sequencing data assembly, splicing, expression measurement, differentially expressed gene screening, WGCNA, and visual analysis of the samples were all performed using the free online Majorbio Cloud Platform (www.majorbio.com). Sequence alignment and phylogenetic tree construction of MYB25-related 


\section{sequences}

The MYB25-related sequences from other species were identified from the NCBI database using PcMYB25 as a bait. Sequence alignment was conducted using DNAMAN with default parameters and a phylogenetic tree was constructed using MEGA 7.0.

\section{Transient overexpression in patchouli leaves}

Patchouli plants with six leaves were used for transient overexpression by agrobacteriamediated transformation. In order to overexpress PcMYB25 in the patchouli leaves, the overexpression vector pJLTRBO (source should be indicated) was used. The 969-bp PcMYB25 ORF was constructed between the NotI and PacI sites. The resulting pJLTRBO-PcMYB25 and an empty pJLTRBO vector (a negative control) were transformed separately into Agrobacterium GV3101 (pSoup p19) (Weidi Biothchnology, Shanghai). Then the transformed agrobacteria were plated on Luria-Bertani plates containing $50 \mu \mathrm{g} / \mathrm{mL}$ kanamycin and $25 \mu \mathrm{g} / \mathrm{mL}$ rifampicin for $48 \mathrm{~h}$ for plasmid selection.

The method of agrobacteria-mediated transient expression has been described previously(Wang et al. 2019b). The agrobacteria were cultured in Luria-Bertani medium supplemented with $50 \mu \mathrm{g} / \mathrm{mL}$ kanamycin and $25 \mu \mathrm{g} / \mathrm{mL}$ rifampicin for $24 \mathrm{~h}$ until the OD600 reached $0.6-0.8$, then the agrobacteria were harvested by centrifugation and resuspended in the

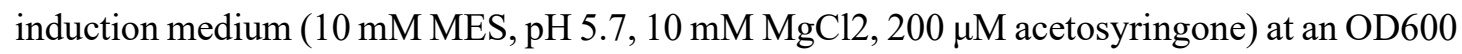
of 1.0. Cells were incubated at room temperature for 2-4 h. Two to three leaves per plant were infiltrated on the abaxial sides using $1 \mathrm{~mL}$ needleless syringe. After infection, the plants were incubated at $25^{\circ} \mathrm{C}$ for $3-5$ days. Then the patchouli leaves were collected and stored at $-80{ }^{\circ} \mathrm{C}$ for RNA extraction and patchouli alcohol content detection.

\section{RNA extraction and quantitative reverse-transcription PCR (RT-qPCR)}

Total RNA was extracted from patchouli leaves using the Favor PrepTM Plant Total RNA Mini Kit (Favor Biothch, China). Then HiScript III RT Super Mix for qPCR (+gDNA wiper) (Vazyme Biotech, China) was used for reverse transcription. For each sample, a $200 \mathrm{mg}$ RNA was used for reverse transcription. AceQ Universal SYBR qPCR Master Mix (Vazyme Biotech, China) was used for qPCR. The internal reference gene 18S rRNA was used to normalize the relative expression for tested genes. The values shown are averages of three biological replicates. The primers used are listed in Additional file 1: Fig. S6. 


\section{PA extraction and quantification}

The method of PA extraction and quantification used has been described previously (Wang et al. 2019b). Patchouli leaves were ground in liquid nitrogen and $200 \mathrm{mg}$ of powder, after adding $1.5 \mathrm{~mL}$ hexane, was extracted by ultrasonic at $60 \mathrm{~Hz}$ for $30 \mathrm{~min}$. Then, the mixture was placed in a $56{ }^{\circ} \mathrm{C}$ water bath for $1 \mathrm{~h}$. After a short centrifugation, the supernatant was filtered with a $0.22 \mu \mathrm{m}$ organic microporous membrane, then the filtrate was taken as the test solution for GC-MS detection.

Agilent 7890B/5977A GC-MS and HP5-ms capillary column $(30 \mathrm{~m} \times 250 \mu \mathrm{m} \times 0.25 \mu \mathrm{m})$ was used for the separation and detection of PA. the procedure was set with an initial temperature of $50^{\circ} \mathrm{C}$ and kept for $2 \mathrm{~min}$, then the temperature is increased to $130^{\circ} \mathrm{C}$ at a rate of $20^{\circ} \mathrm{C} / \mathrm{min}$, and the temperature is increased to $150^{\circ} \mathrm{C}$ at $2{ }^{\circ} \mathrm{C} / \mathrm{min}$, and maintained for $5 \mathrm{~min}$ at $150{ }^{\circ} \mathrm{C}$. After that, the temperature increased to $230^{\circ} \mathrm{C}$ at rate of $20^{\circ} \mathrm{C} / \mathrm{min}$. In addition, the patchouli alcohol purchased from Feiyu (Nantong, China) was used as a standard.

\section{Results}

\section{PA content altered in patchouli leaves treated with various hormones}

The biosynthesis of PA mainly occurs in the leaves of patchouli and the content of secondary metabolites is often affected by exogenous plant hormones (Chen et al. 2014; Santner and Estelle 2009; Verma et al. 2019). The six-leaf-stage patchouli leaves were treated with $0.5 \%$ ethanol (control) or various hormones (MeJA, SA, ETH, ABA, and MeJA/ETH (MandE)). Among different hormone treatments, the highest PA content was found with the MeJA, and the lowest PA content was observed with MandE. The PA content observed with the MeJA showed significantly difference from that observed with the SA, ETH, and MandE (Additional file 1: Fig. S1).

\section{RNA-sequencing, assembly of sequences and functional annotation}

To find the critical transcription factors involved in regulation of the biosynthetic pathway of patchouli alcohol, patchouli leaves in triplicate were sprayed by $0.5 \%$ ethanol (control), MeJA, ETH, ABA, SA, and MandE, respectively. A total of 154,779 unigenes were assembled, with a size of $223,580,816 \mathrm{bp}$ and an N50 of 1058 . These data indicate that the quality of RNA sequencing is high enough to be used for further analysis. To access the expression pattern of 
the patchouli transcriptomes for various hormone treatments, the assembled transcriptome sequences were annotated and compared with six databases: NR, Swiss-Prot, Pfam, COG, GO and KEGG. Of total 154,779 unigenes annotated: 60,039, 55,123, and 43,196 unigenes were annotated from NR, Swiss-Prot, and Pfam, respectively. We used GO, COG, and KEGG databases to classify unigenes. In order to understand the function and classification of unigenes in a global view, GO functional annotations were performed. Of 43,477 unigenes annotated to this database, three main categories were grouped: biological process (BP), cellular component (CC), and molecular function (MF) (Additional file 1: Fig. S2). For biological process, organic substance metabolic process (GO:0071704), primary metabolic process (GO:0044238), cellular metabolic process (GO:0044237) were the most enriched items. For cellular component, unigenes related to membrane part (GO:0044425), cell part (GO:0044464), intrinsic component of membrane (GO:0031224) are the most abundant items. For molecular function category, organic cyclic compound binding (GO:0097159), heterocyclic compound binding (GO:1901363), and ion binding (GO:0043167) were the most highly represented GO terms. Of 8,162 unigenes annotated with COG, functional groups belong to 24 categories (Additional file 1: Fig. S3): 758 unigenes belong to Translation, ribosomal structure and biogenesis (the $\mathrm{J}$ category). Moreover, 35,368 unigenes were mapped into the KEGG pathways. The most representative pathways were "translation", "Carbohydrate metabolism" and "Folding, sorting and degradation" (Additional file 1: Fig. S4).

\section{Identification of differentially expressed genes (DEGs)}

To find the potential transcription factors involved in regulation of the PA biosynthetic pathway, we identified DEGs among the five hormone treatment groups by comparing MandE, MeJA, SA, ABA, and ETH with control; and by comparing MandE with ETH or MeJA. Here, DESeq2 was used for differential expression analysis, and genes with $\mathrm{p}<0.05$ and $\mathrm{FC} \geq 2$ or $\mathrm{FC} \leq 0.5$ (genes up-regulated twice or down-regulated more than two times) were considered DEGs. Here, we found a total of 14,419 DEGs. The numbers of up- and down-regulated DEGs of all comparisons were shown in Additional file 1: Fig. S5. Among them, compared with leaf, MandE has the most differentially expressed genes after treatment, with a total of 9563 unigenes. Among them, there are 5086 up-regulated genes and 4477 down-regulated genes. Compared 
with control, SA has the least up-regulated and down-regulated unigenes (77 and 54). GO and KEGG enrichment analysis were performed on DEGs to predict their biological functions. The GO enrichment analysis divided 14,419 differentially expressed unigenes into three categories: cellular component, molecular function, biological process (Fig. 1A). Among the cell components, membrane (19.60\%) and membrane part (18.98\%) accounted for the highest proportions. Among molecular functions, catalysis and binding account for the highest proportions, at $30 \%$ and $26.87 \%$. The most enriched items are membrane $(19.60 \%)$ and membrane part (18.98\%) for cellular component category; catalysis $(30 \%)$ and binding (26.87\%) for molecular function category; and metabolic process (17.22\%) and cellular process (16.57\%) for biological process category. The results reflected that more DEGs are involved in metabolic processes or cellular processes and have catalytic and binding functions. By KEGG pathway analysis, it was found that 151 unigenes participated in plant hormone signal transduction, 46 unigenes participated in terpenoid backbone biosynthesis, and 34 unigenes participated in sesquiterpene and triterpenoid biosynthesis (Fig. 1B).

\section{Construction of the WGCNA and the identification of hub genes}

The DEGs were evaluated using WGCNA. Here, genes whose expression level is less than one and whose coefficient of variation is less than 0.1 are filtered. Then, the soft power is adjusted to 12, and the module are identified. Here, the processed genes are divided into 10 modules (Fig. 2A). Association of the PA content (trait) with 10 modules was analyzed to identified key modules and potentially involved transcription factors (Fig. 2B). Among these 10 modules, four modules, MEmagenta $\left(\mathrm{r}^{2}=-0.643, p\right.$-value $\left.=0.004\right)$, MEgreen $\left(\mathrm{r}^{2}=-0.591, p\right.$ value $=0.0098)$, MEpink $\left(\mathrm{r}^{2}=0.544, p\right.$-value $\left.=0.0196\right)$, and MEblack $\left(\mathrm{r}^{2}=-0.525, p\right.$-value $\left.=0.0253\right)$, were regarded as the key modules associated with the patchouli alcohol content $(\mathrm{P}<0.05)$ (Fig. 2B). Notably, MEmagenta $\left(\mathrm{r}^{2}=-0.643, p\right.$-value $\left.=0.004\right)$ has the strongest correction to patchouli alcohol among these 10 modules, but there was not any transcription factor being found in this module. Therefore, we focus on analysis of MEgreen, which is significantly related to the content of patchouli alcohol $\left(\mathrm{r}^{2}=-0.591, p\right.$-value $\left.=0.0098\right)$. A total of 358 genes are included in this module, including 8 structural genes related to patchouli alcohol synthesis (Additional file: fig: S 9). In addition, 14 out of the 358 genes have been identified as transcription factors (Additional file: fig: S8). Especially, we found a key transcription factor, 
TRINITY_DN73869_c4_g1 (kME=0.89181), name here as PcMYB25. As a crucial gene, PcMYB25 processes top 30 degree in MEgreen module (degree=44.12) (Additional file: fig: S7). Moreover, the gene expression correlation analysis network diagram shows that $P c M Y B 25$ is closely associated with terpenoid metabolism-related genes (Fig. 2C). In addition, according to previous reports, MYB is widely involved in the process of plant secondary metabolism (Dubos et al. 2010). Thus, we speculate that PcMYB25 may play an essential role in patchouli alcohol biosynthesis.

\section{Overexpression of PcMYB25 increased the PcPTS expression level and patchouli alcohol} production

To reveal the function of PCMYB25 in patchouli, the pJLTRBO vector was used to overexpress PcMYB25 in the leaves of patchouli, which is mediated by agrobacteria (Fig. 3A). The efficiency of this overexpression was evaluated by RT-qPCR four days after the injection. Compared with that of the control group, the expression level in the experimental groups increased by 2.55 -fold (Fig. 3B). The transcript levels of some genes in the upstream patchouli alcohol biosynthetic pathway, such as $A A C T$ and $P M K$, were significantly increased; on the contrary, the HMGR level was considerably reduced. Notably, the expression level of PcPTS was significantly increased, by $309 \%$, compared with that of the control group (Fig. 4). Interestingly, the PA content was increased by $85 \%$ in patchouli leaves overexpressing PcMYB25, which is in accordance with the upregulation of PcPTS (Fig. 3C and Fig. 3D).

\section{PcMYB25 codes a R2R3 MYB protein}

We cloned the full-length coding sequence of PcMYB25 from patchouli cDNAs. After sequence analysis, the ORF including the stop codon of PcMYB25 was determined to be 969 bp in length and encodes 322 aa. Amino acid sequence alignment confirms that PcMYB25 shares high similarities with the PcMYB25-related proteins retrieved from Rehmannia glutinosa, Salvia splendens, and Actinidia rufa. PcMYB25 harbors the conserved R2 and R3 domains in N-terminus, typical of a R2R3-MYB transcription factor. To help predicting potential functions for PcMYB25, a phylogenetic tree was constructed by MEGA 7 (Fig. 5B). Results showed that the closest MYB proteins were from Rehmannia glutinosa, Salvia splendens and Salvia miltiorrhiza. PcMYB25 also displays high similarity (95\%) to the R2-R3 
conserved domains with Arabidopsis AtMYB25 (Fig. 5B).

\section{Discussion}

\section{Changes of patchouli under different hormone treatments}

Plants are usually subjected to various adversity stresses during their growth. These adverse stresses usually lead to changes in plant hormone levels, thereby affecting the synthesis and accumulation of secondary metabolites (Santner and Estelle 2009). Like most secondary metabolites, the synthesis of patchouli alcohol has previously been found to be associated with phytohormone. Tang et al used MeJA, ABA, SA to treat the leaves of patchouli, it was found that the expression of HMGS, PMV and MVK on the MVA pathway have increased (Tang et al. 2019). In comparison with control, content of secondary metabolites including PA were found to be significantly different in patchouli leaves treated with MeJA, ETH, or MandE. After treatments, 254, 229, and 400, respectively, differentially expressed proteins were identified. These differentially expressed proteins are mainly involved in photosynthesis, secondary metabolites, carbohydrate and energy metabolism ( $\mathrm{Li}$ et al. 2019). These previous studies have shown expression levels or content of genes, proteins and secondary metabolites changed when patchouli leaves were treated with exogenous hormones. To determine the effect of various hormones on PA content, PA content of patchouli leaves treated with various hormones was determined. The highest content of PA was found with MeJA-treated leaves, and the lowest content was associated with MandE-treated. The PA content of the MeJA-treated leaves was significantly different from that of the SA-, ETH-, and MandE-treated.

RNA-Seq is a powerful tool to identify the transcriptional regulation mechanism of secondary metabolism. This study provides a transcriptome survey to understand the functions of DEGs. DEGs identified are mainly enriched in metabolic and cellular processes, and are mainly involved in catalysis and binding. The enriched signal pathways include plant hormone signal transduction, MAPK signaling pathway, and alpha-linoenic acid metabolism pathways.

\section{Co-expression facilitates the identification of PA-related transcription factors}

The MYB proteins belong to a large, functionally diverse family of transcription factors in all eukaryotes, and participate in cell proliferation (Yusenko et al. 2020), differentiation (Jakoby et al. 2008), apoptosis (Srivastava et al. 2015), abiotic and biotic stress (Kim et al. 2013; Shen 
et al. 2017) and secondary metabolism (Matías-Hernández et al. 2017; Paz Ares et al. 1987). The N-termini of the MYB transcription factors contain 1-3 conserved DNA binding domains: R1, R2, R3, and/or R4. Based on the presence of specific DNA binding domains, MYB transcription factors can be divided into four categories: R1-MYBs, R2R3-MYBs, R1R2R3MYBs, and R4-MYBs. According to previous reports, R2R3-MYBs are transcription regulators for synthesizing secondary metabolites in plants. For example, PpMYB140 works as a transcriptional repressor to directly inhibit the expression of anthocyanin-related genes and prevent anthocyanin over accumulation in bananas (Ni et al. 2021). CaMYB48 encodes an R2R3 transcription factor to activate the promoters of AT3 and KasIa, leading to increases in Cap and DhCap (Sun et al. 2020). Using WGCNA, we identified a R2R3-MYB, PcMYB25, which is most similar to an R2R3-MYB protein from Rehmannia glutinosa. Available studies on PcMYB25-related transcription factors indicated roles on plant cell growth and cotton fiber formation. Wang et al. identified a MYB factor from Rehmannia glutinosa as a MYB25 for the first time, however, functions of this MYB had not been illuminated in detail (Wang et al. 2015). An Arabidopsis T-DNA-inserted MYB25-null mutant exhibited deformed pollen cell wall, twocelled pollen, and misarranged male germ unit, indicating a role of AtMYB25 in Arabidopsis pollen development (Reňák et al. 2012). An Arabidopsis T-DNA-inserted MYB25-null mutant exhibited deformed pollen cell wall, two-celled pollen, and misarranged male germ unit, indicating a role of AtMYB25 in Arabidopsis pollen development (Walford et al. 2011). Our work on PcMYB25, however, is the first study showing involvement of a MYB25 in secondary metabolism. By WGCNA, we identified a MEgreen module, which shows a tight correlation between gene expression patterns and PA content. Numerous genes from this module were related to terpene synthesis. Prior to this study, the co-expression network had not been used to identify genes and transcription factors related to PA biosynthesis. Although, genes involved in the PA biosynthetic pathway have been identified, the regulatory network response has not been fully elucidated. Identifying more critical genes from this module and their interplays will be a topic in the future research to further examine regulatory network for PA biosynthesis.

In the combined correlation between the expression level of PcMYB25 and patchouli alcohol content, the Pearson correlation coefficient was 0.926. Similarly, the correlation coefficients of PcMYB25 and PTS expression was 0.897 . Thus, we speculate that PcMYB25 might be a key 
activator to enhance biosynthesis of patchouli alcohol in its leaves. In addition, after the overexpression of PcMYB25, the PA content in plant leaves was increased by $85 \%$. After testing the gene expression in the patchouli alcohol synthetic pathway, it was found that PcMYB25 had a negligible effect on the upstream genes of the pathway, but it specifically increased the expression of PTS, which was 4.09-fold greater than the original expression level. However, module and trait correlation heat map show a negative correction ( $\mathrm{r} 2=-0.591, p$-value $=0.0098)$ for green module with PA (Fig. 2B). A similar situation exists in Pan's research (Pan et al. 2020). Pan et al. performed a WGCNA of all genes of Y12-4 (cold-tolerance genotypes). Modulestrait relation showed that $L T G 5$ in the module greenyellow $(\mathrm{r} 2=0.35$, $\mathrm{p}$-value $=0.2)$ is a key gene in regulating cold tolerance at the germination stage. Overexpression of LTG5 can increase the germination rate of rice at low temperature. However, there are COLDl (Ma et al. 2015), CTB4a (Zhang et al. 2017), LTG1 (Lu et al. 2014), ctb1 (Saito et al. 2010), ICE1 (Chinnusamy et al. 2007) in the blue module ( $\mathrm{r} 2=-0.98, p$-value $\left.=2 \mathrm{e}^{-10}\right), q L T G 3-1$ (Fujino and Iwata 2011) in the brown module $\left(\mathrm{r} 2=-0.92\right.$, $p$-value $\left.=2 \mathrm{e}^{-0.6}\right)$. These genes can improve the tolerance of rice at low temperature conditions as well.

In addition, constructing a co-expression network, the correlation between gene expression level and trait is considered, but the influence of gene interaction on trait is not considered. Modules clustering heat map showed that, genes in the green module highly expressed in MandE treatment group, lowly expressed in ETH treatment group, and expressed irregularly in other treatment groups. However, the PA content was the lowest under the MandE treatment, so the WGCNA analysis showed that the module was negatively correlated with the gene. In addition, the module is negatively correlated with genes, but there may be differences of genes in green module, which could be the reason of the positive correlation between the PcMYB25 and PA.

\section{Conclusion}

In this study, through WGCNA, we identified that the MEgreen module was related to patchouli alcohol biosynthesis. This module provides candidate genes related to patchouli alcohol, and the hub transcription factor PcMYB25 was able to upregulate PTS to increase the content of patchouli alcohol. This is the first time that MYB25 has been reported as a transcriptional activator to regulate the biosynthesis of secondary metabolism. This discovery 
lays the foundation for further research on the transcriptional regulatory network of patchouli alcohol biosynthesis in patchouli and provides mechanical insights into the evolution of plant secondary metabolism.

390

\section{1}

392

393

394

395

396

397

398

399

400

401

402

403

404

405

406

407

408

409

410

411

412

413

414

415

416

417

418

419

420

421

422

423

424

425

426

427

428

\section{Abbreviations}

AACT: acetoacetyl-CoA thiolase

ABA: abscisic acid

AS: anthranilic acid.

BP: biological process

Cap: capsaicinoid

CC: cellular component

COG: Clusters of Orthologous Groups of Proteins

CPR: cytochrome $\mathrm{P} 450$ reductase

D4H: desacetoxyvindoline 4-hydroxylase

DEG: differentially expressed genes

DMAPP: dimethylallylpyrophosphate

DXS: 1-deoxy-D-xylulose 5-phosphate synthase

\section{ETH: ethrel}

FPP: synthesis farnesyl diphosphate

FPPS: farnesyl pyrophosphate synthase

G10H: geraniol 10hydroxylase

GC-MS: gas chromatography-mass spectrometry

GO: Gene Ontology

HMGR: 3-hydroxy-3-methylglutaryl-coenzyme A reductase

HMGS: hydroxy-3-methylglutaryl-coenzyme A synthase

IPP: isopentenyl pyrophosphate

IPPI: isopentenyl diphosphate

JA: methyl jasmonate

KEGG: Kyoto Encyclopedia of Genes and Genomes

MandE: MeJA with ETH

MeJA: methyl jasmonate

MF: molecular function

MVA pathway: mevalonic acid pathway

MVA: mevalonate acid

MVD: mevalonate diphosphate decarboxylase

MVK: mevalonate kinase

NR: non-redundant protein sequence database

PA: patchouli alcohol

PMK: phosphomevalonate kinase

PTS: patchouli alcohol synthase

RT-qPCR: quantitative real-time PCR

SA: salicylic acid 
SGD: strictosidine beta-glucosidase

430 SLS: secologanin synthase

431 STR: strictosidine synthase

432 Swiss-Prot: Swiss-Prot Protein Sequence Database

433 TDC: tryptophan decarboxylase

434 TIA: terpenoid indole alkaloids

435 WGCNA: Weighted gene co-expression correlation network analysis

436 Y2H: yeast two-hybrid assay

\section{Declarations}

\section{Authors' contributions}

440 RTZ and LKC designed the study; XXZ and XLW performed experiments; HLH and DDW

441 analyzed the data; XBW, JRL, and XZC helped the field works; XXZ wrote the manuscript.

442 LKC edited the manuscript and provided guidance during this experimentation. All authors read

443 and approved the final manuscript.

\section{Competing interests}

445 The authors declare that they have no conflicts of interest.

\section{Consent for publication}

$447 \quad$ Not applicable.

\section{Ethics approval and consent to participate}

449 Not applicable. All applicable international, national, and/or institutional guidelines for the care

450 and use of animals were followed.

\section{$451 \quad$ Funding}

452 This research was funded by the Natural Science Foundation of Guangdong Province (No. 2019A1515011542), National Natural Science Foundation of China (No. 81803657), Project of south medicine innovation team in modern agricultural industry technology system of

455 Guangdong Province (No. 2019KJ148).

458 Fig.1 A. GO function classification of DEGs. The ordinate on the left of the figure represents the secondary classification of GO, the abscissa represents the number of unigenes included in the 
secondary classification, and the three colors on the right represent the three major branches of GO (BP, CC, MF). B. KEGG classification of DEGs. The ordinate shows the names of the KEGG metabolic pathways and the abscissa indicates the number of unigenes.

Fig. 2 Network analysis dendrogram shows the modules identified by Weighted Gene Coexpression Network Analysis (WGCNA). A. Tree diagram with color annotations. Each color represents a module. B. Module and phenotype correlation heat map. Module-patchouli alcohol weight correlation and corresponding $P$-value. The left panel shows 10 modules. The color scale on the right shows the correlation of module characteristics from -1 (blue) to 1 (red). C. Network analysis of PcMYB25, genes related with terpenoid and other genes in MEgreen module. Red pot represents $P c M Y B 25$, orange spots represent terpenoid related genes, blue spots represent other 358 genes in MEgreen.

Fig. 3 Transient overexpress analysis of PcMYB25 in patchouli leaves. A. PcMYB25 was cloned into overexpression vector pJLTRBO to form pJLTRBO-PcMYB25 construct with the restriction enzyme site PacI and NotI. B. The expression level of PcMYB25 in control (control group) and OX (overexpression group) analyzed by RT-qPCR. C. Chromatograms for PA content in control and PcMYB25-overexpressed leaves in comparison with standard. D. The content of patchouli alcohol detected in control and OX. Asterisks indicate a significant difference from the control (Student's ttest; $* * * \mathrm{p}<0.001, * \mathrm{p}<0.05)$. FW, fresh weight.

Fig. 4 The expression level of genes on the patchoulol synthesis pathway after transient overexpression of PcMYB25. The expression level of genes on MVA pathway was determined by RT-qPCR, and control represents an empty vector control. Using Pc18S as the internal reference gene, the relative expression level of the gene was calculated according to the 2- $\triangle \Delta \mathrm{Ct}$ method. Asterisks represent significant differences $\left({ }^{*} \mathrm{P}<0.05,{ }^{*} \mathrm{P}<0.01\right)$.

Fig. 5 Phylogenetic analysis of PcMYB25. A. Sequence analysis of PcMYB25 and other MYB transcription factors. In order to compare the MYB sequence between different plant species, PcMYB25 was used as a bait gene, and MYB transcription factors with similar sequences in Rehmannia glutinosa, Salvia miltiorrhiza, Actinidia violacea and Arabidopsis thaliana were identified from NCBI database. B. Phylogenetic tree of MYB transcription factors. Some MYB protein share similar sequences for phylogenetic analysis. A phylogenetic tree was constructed by 
491

492

493

494

495

496

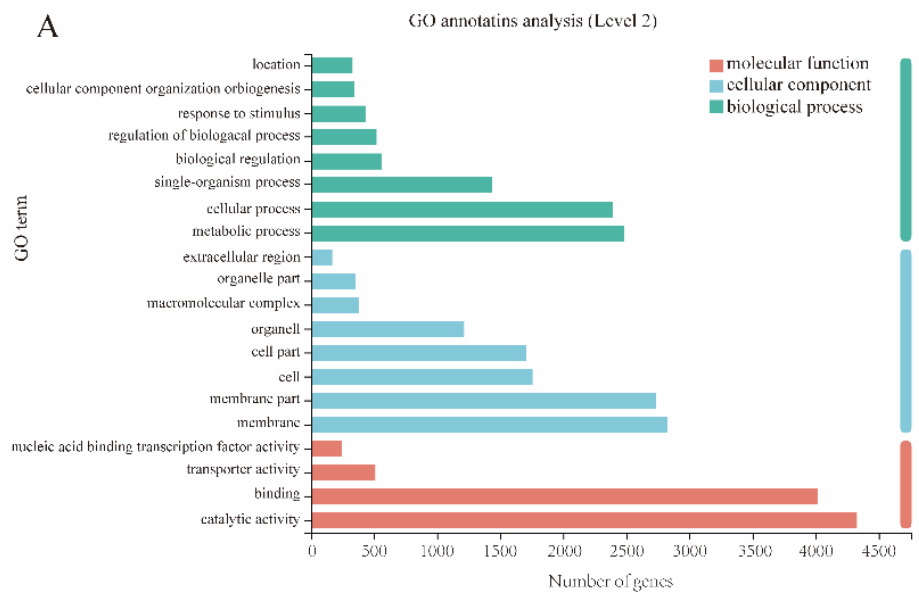

$$
\text { B }
$$

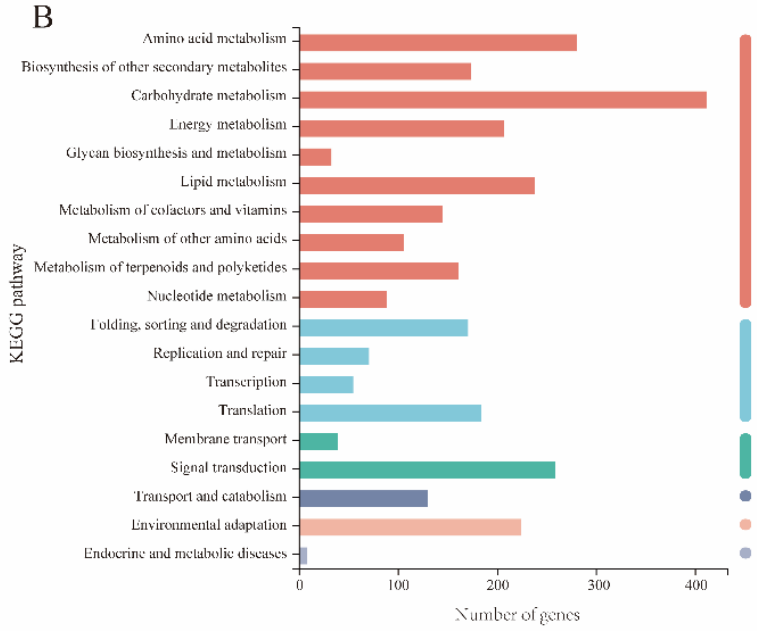

Metabolism

Genetix Information Processing

F.nvironmental Information Processing

Cellular Processes

Human Distass:

497

498

499

500

501

502 

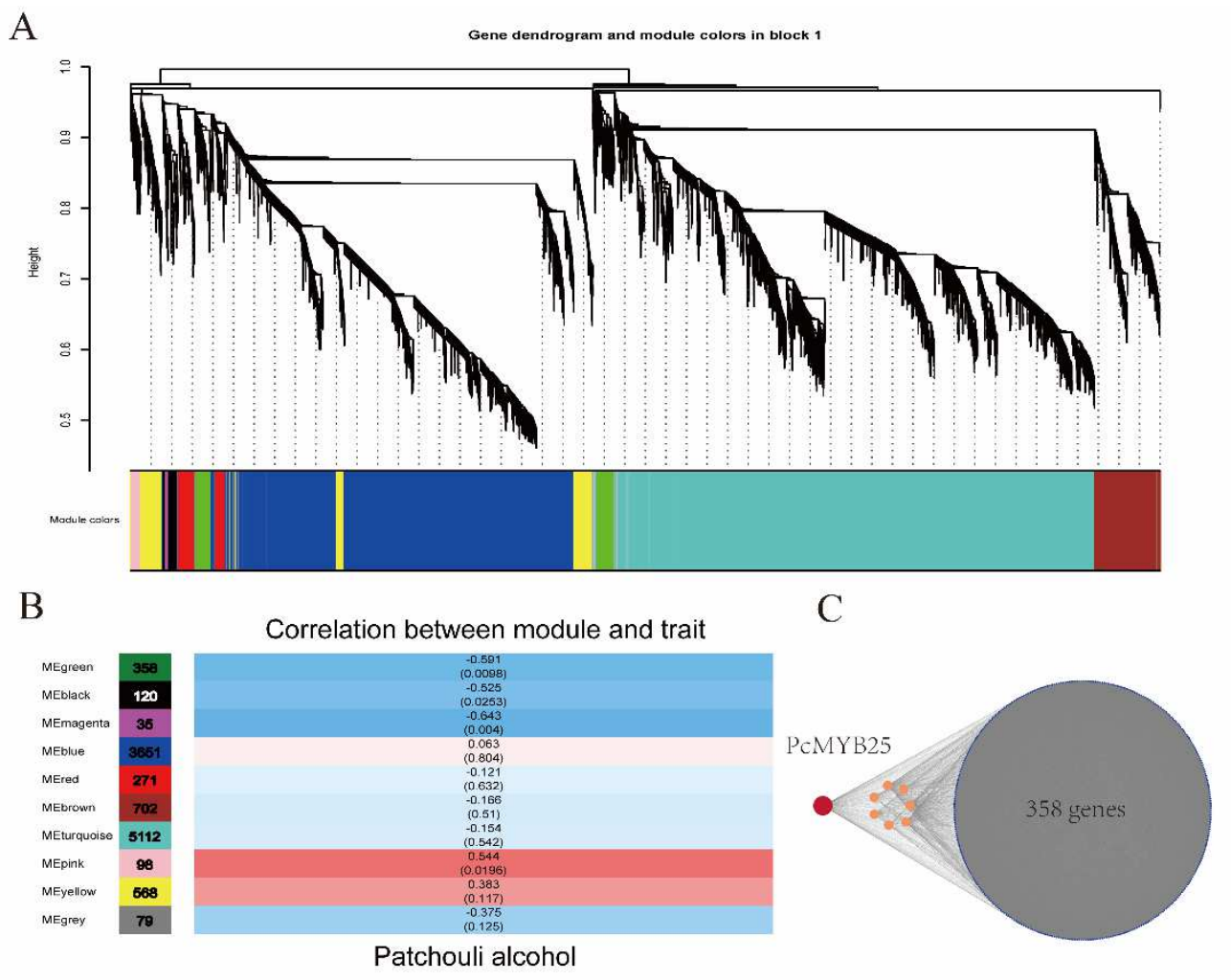

A

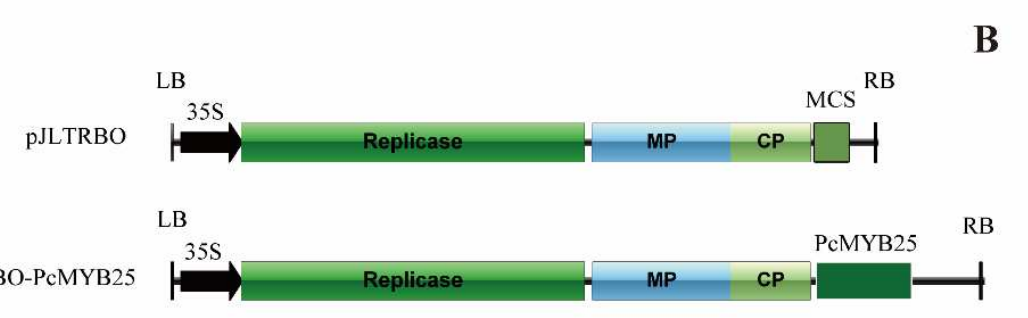

pJLTRBO-PcMYB25

Replicase

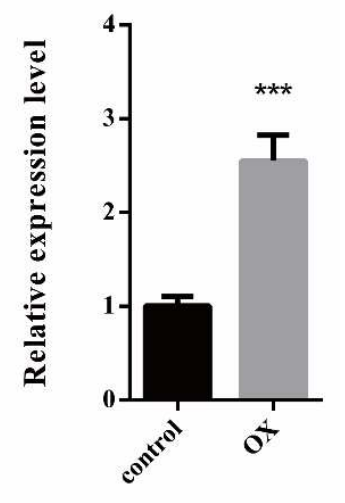

C
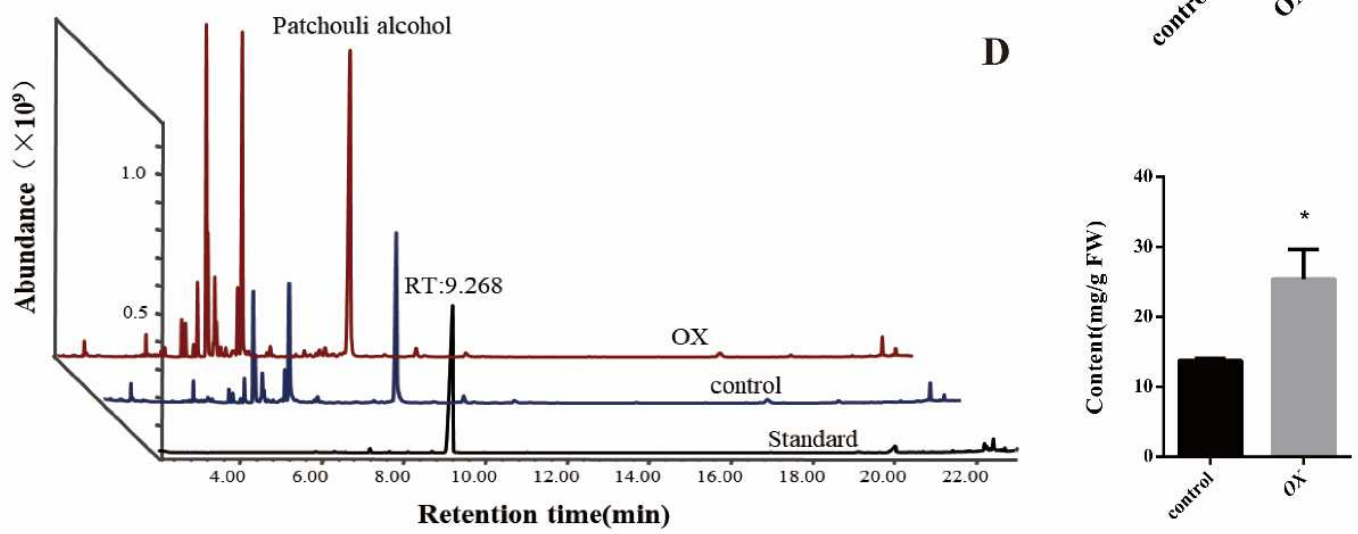


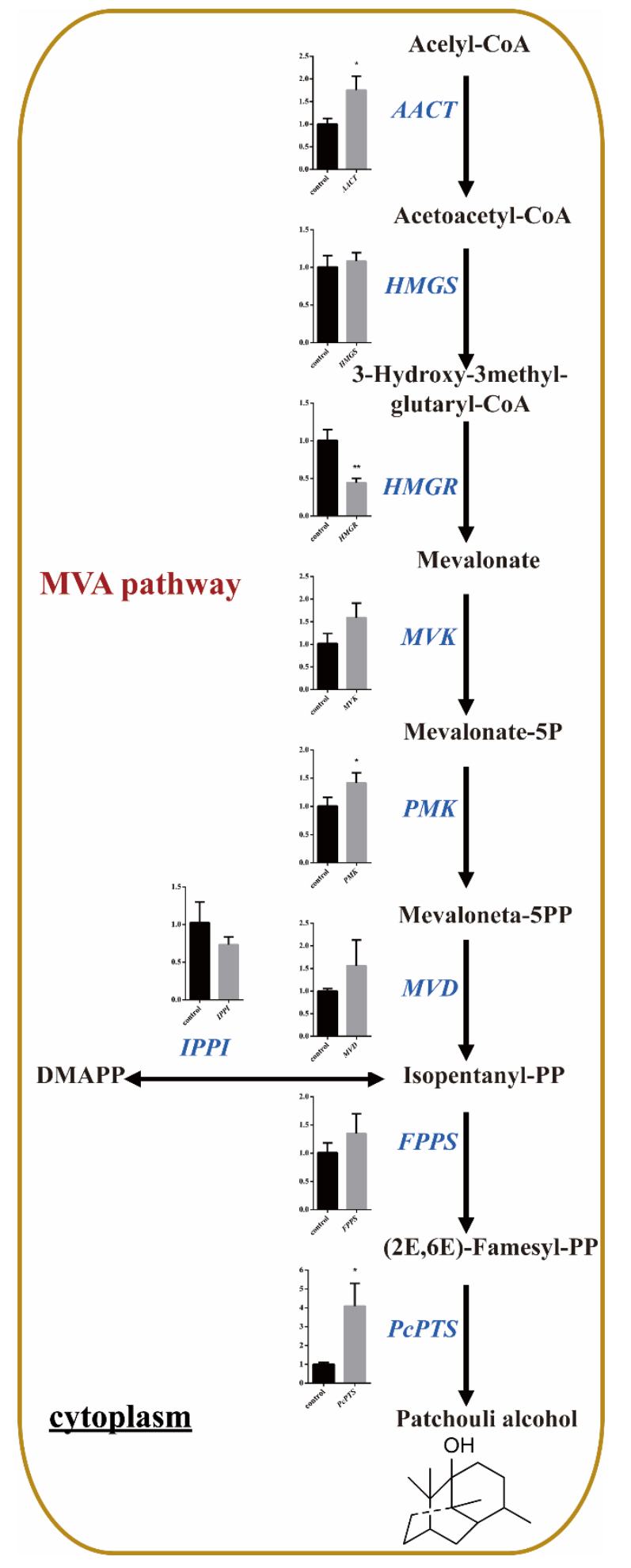




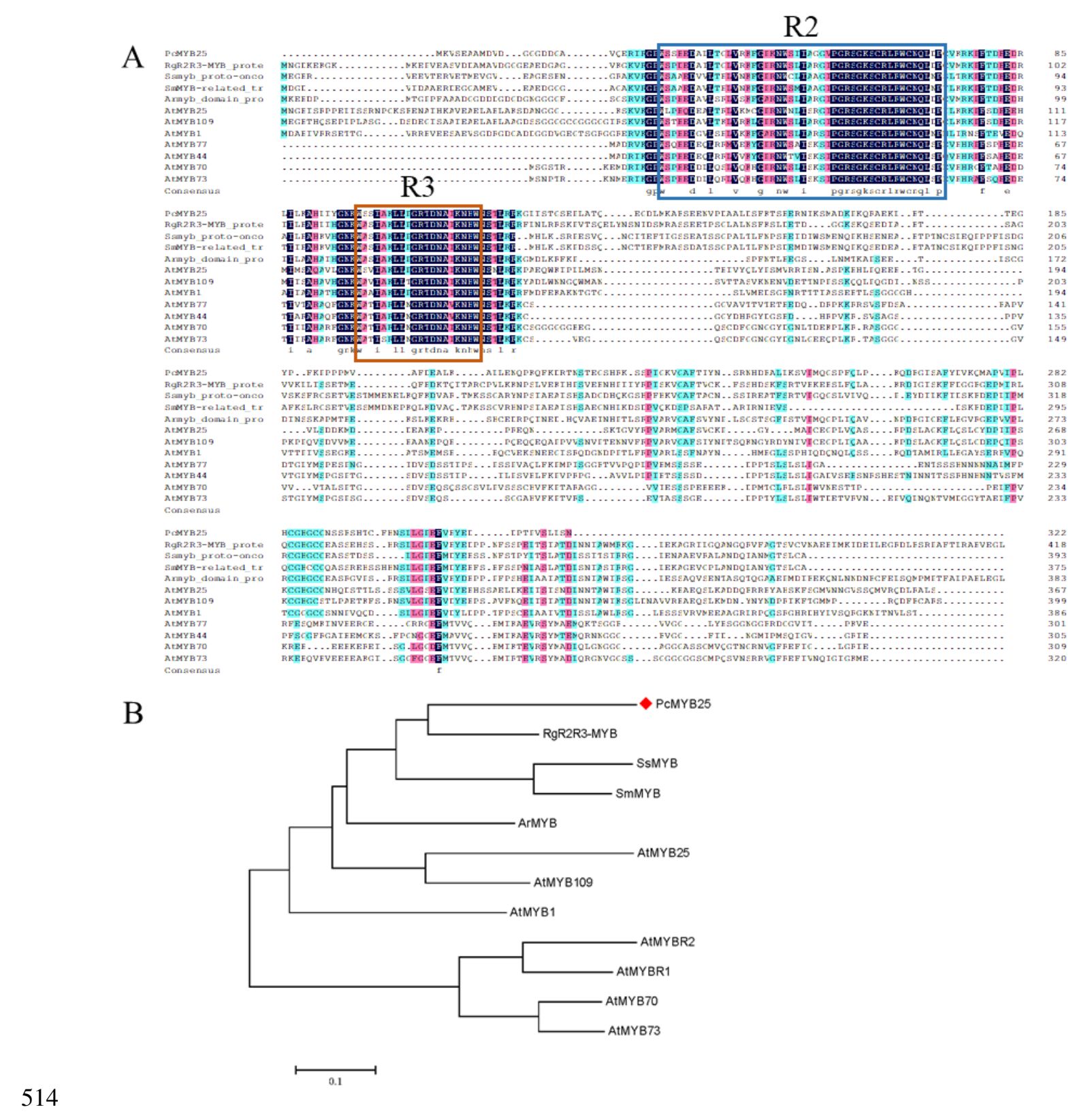

\section{References:}

516 Bouvier F, Rahier A, Camara B (2005) Biogenesis, molecular regulation and function of plant 517 isoprenoids Prog Lipid Res 44:357-429 doi:10.1016/j.plipres.2005.09.003

518 Buchner O et al. (2017) Drought affects the heat-hardening capacity of alpine plants as indicated by 519 changes in xanthophyll cycle pigments, singlet oxygen scavenging, a -tocopherol and plant hormones 520 Environ Exp Bot 133:159-175 doi:10.1016/j.envexpbot.2016.10.010

521 Chen X et al. (2020) PatSWC4, a methyl jasmonate-responsive MYB (v-myb avian myeloblastosis viral 522 oncogene homolog)-related transcription factor, positively regulates patchoulol biosynthesis in 523 Pogostemon cablin Ind Crop Prod 154:112672 doi:10.1016/j.indcrop.2020.112672

524 Chen Y, Wu Y, Xu Y, Zhang J, Song X, Zhu G, Hu X (2014) Dynamic accumulation of sesquiterpenes 525 in essential oil of Pogostemon cablin Revista Brasileira de Farmacognosia 24:626-634 526 doi:10.1016/j.bjp.2014.11.001 
Chinnusamy V, Zhu J, Zhu J (2007) Cold stress regulation of gene expression in plants Trends Plant Sci 12:444-451 doi:10.1016/j.tplants.2007.07.002

Deguerry F, Pastore L, Wu S, Clark A, Chappell J, Schalk M (2006) The diverse sesquiterpene profile of patchouli, Pogostemon cablin, is correlated with a limited number of sesquiterpene synthases Arch Biochem Biophys 454:123-136 doi:10.1016/j.abb.2006.08.006

Dubos C, Stracke R, Grotewold E, Weisshaar B, Martin C, Lepiniec L (2010) MYB transcription factors in Arabidopsis Trends Plant Sci 15:573-581 doi:10.1016/j.tplants.2010.06.005

El-Sharkawy I, Liang D, Xu K (2015) Transcriptome analysis of an apple (Malus xdomestica) yellow fruit somatic mutation identifies a gene network module highly associated with anthocyanin and epigenetic regulation J Exp Bot 66:7359-7376 doi:10.1093/jxb/erv433

Fujino K, Iwata N (2011) Selection for low-temperature germinability on the short arm of chromosome 3 in rice cultivars adapted to Hokkaido, Japan Theor Appl Genet 123:1089-1097 doi:10.1007/s00122011-1650-4

Jakoby MJ et al. (2008) Transcriptional Profiling of Mature Arabidopsis Trichomes Reveals That NOECK Encodes the MIXTA-like Transcriptional Regulator MYB106 Plant physiology (Bethesda) 148:1583-1602 doi:10.1104/pp.108.126979

Jan S, Abbas N, Ashraf M, Ahmad P (2019) Roles of potential plant hormones and transcription factors in controlling leaf senescence and drought tolerance Protoplasma 256:313-329 doi:10.1007/s00709-0181310-5

Kim JH, Nguyen NH, Jeong CY, Nguyen NT, Hong S, Lee H (2013) Loss of the R2R3 MYB, AtMyb73, causes hyper-induction of the SOS1 and SOS3 genes in response to high salinity in Arabidopsis J Plant Physiol 170:1461-1465 doi:10.1016/j.jplph.2013.05.011

Lee H, Lee J, Smolensky D, Lee S (2020) Potential benefits of patchouli alcohol in prevention of human diseases: A mechanistic review Int Immunopharmacol 89:107056 doi:10.1016/j.intimp.2020.107056

Li J et al. (2019) Comparative iTRAQ-based proteomic analysis provides insight into a complex regulatory network of Pogostemon cablin in response to exogenous MeJA and Ethrel Ind Crop Prod 140:111661 doi:10.1016/j.indcrop.2019.111661

Liao P, Hemmerlin A, Bach TJ, Chye M (2016) The potential of the mevalonate pathway for enhanced isoprenoid production Biotechnol Adv 34:697-713 doi:10.1016/j.biotechadv.2016.03.005

Lu C, Pu Y, Liu Y, Li Y, Qu J, Huang H, Dai S (2019) Comparative transcriptomics and weighted gene co-expression correlation network analysis (WGCNA) reveal potential regulation mechanism of carotenoid accumulation in Chrysanthemum x morifolium Plant Physiol Biochem 142:415-428 doi:10.1016/j.plaphy.2019.07.023

Lu G et al. (2014) Rice LTG1 is involved in adaptive growth and fitness under low ambient temperature The Plant Journal 78:468-480 doi:10.1111/tpj.12487

Lu X et al. (2013) AaORA, a trichome-specific AP2/ERF transcription factor ofArtemisia annua, is a positive regulator in the artemisinin biosynthetic pathway and in disease resistance toBotrytis cinerea New Phytol 198:1191-1202 doi:10.1111/nph.12207

Ma Y et al. (2015) COLD1 confers chilling tolerance in rice Cell 160:1209-1221 doi:10.1016/j.cell.2015.01.046

Matías-Hernández L, Jiang W, Yang K, Tang K, Brodelius PE, Pelaz S (2017) AaMYB1 and its orthologue AtMYB61 affect terpene metabolism and trichome development inArtemisia annua and Arabidopsis thaliana The Plant Journal 90:520-534 doi:10.1111/tpj.13509

Mitsui R, Nishikawa R, Yamada R, Matsumoto T, Ogino H (2020) Construction of yeast producing 
patchoulol by global metabolic engineering strategy Biotechnol Bioeng 117:1348-1356 doi:10.1002/bit.27284

Ni J, Premathilake AT, Gao Y, Yu W, Tao R, Teng Y, Bai S (2021) Ethylene-activated PpERF105 induces the expression of the repressor-type R2R3-MYB gene PpMYB140 to inhibit anthocyanin biosynthesis in red pear fruit Plant J 105:167-181 doi:10.1111/tpj.15049

Pan Y et al. (2020) Transcriptomic profiling of germinating seeds under cold stress and characterization of the cold-tolerant gene LTG5 in rice Bmc Plant Biol 20 doi:10.1186/s12870-020-02569-Z

Paz Ares J, Ghosal D, Wienand U, Peterson PA, Saedler H (1987) The regulatory c1 locus of Zea mays encodes a protein with homology to myb proto - oncogene products and with structural similarities to transcriptional activators The EMBO journal 6:3553-3558 doi:10.1002/j.1460-2075.1987.tb02684.x

Reňák D, Dupl Áková N, Honys D (2012) Wide-scale screening of T-DNA lines for transcription factor genes affecting male gametophyte development in Arabidopsis Sexual Plant Reproduction 25:39-60 doi:10.1007/s00497-011-0178-8

Saito K, Hayano-Saito Y, Kuroki M, Sato Y (2010) Map-based cloning of the rice cold tolerance gene Ctb1 Plant Sci 179:97-102 doi:10.1016/j.plantsci.2010.04.004

Santner A, Estelle M (2009) Recent advances and emerging trends in plant hormone signalling Nature 459:1071-1078 doi:10.1038/nature08122

Shen Q, Yan T, Fu X, Tang K (2016) Transcriptional regulation of artemisinin biosynthesis in Artemisia annua L. Sci Bull 61:18-25 doi:10.1007/s11434-015-0983-9

Shen X, Guo X, Guo X, Zhao D, Zhao W, Chen J, Li T (2017) PacMYBA, a sweet cherry R2R3-MYB transcription factor, is a positive regulator of salt stress tolerance and pathogen resistance Plant Physiol Bioch 112:302-311 doi:10.1016/j.plaphy.2017.01.015

Srivastava SK et al. (2015) MYB is a novel regulator of pancreatic tumour growth and metastasis Brit J Cancer 113:1694-1703 doi:10.1038/bjc.2015.400

Sun B et al. (2020) Coexpression network analysis reveals an MYB transcriptional activator involved in capsaicinoid biosynthesis in hot peppers Hortic Res-England 7:162 doi:10.1038/s41438-020-00381-2 Swamy MK, Sinniah UR (2016) Patchouli (Pogostemon cablin Benth.): Botany, agrotechnology and biotechnological aspects Ind Crop Prod 87:161-176 doi:10.1016/j.indcrop.2016.04.032

Tang Y, Zhong L, Wang X, Zheng H, Chen L (2019) Molecular identification and expression of sesquiterpene pathway genes responsible for patchoulol biosynthesis and regulation in Pogostemon cablin Bot Stud 60 doi:10.1186/s40529-019-0259-9

Verma RS, Padalia RC, Chauhan A, Singh VR (2019) Chemical composition of leaves, inflorescence, whole aerial-parts and root essential oils of patchouli \{Pogostemon cablin (Blanco) Benth.\} J Essent Oil Res 31:319-325 doi:10.1080/10412905.2019.1566100

Verma V, Ravindran P, Kumar PP (2016) Plant hormone-mediated regulation of stress responses Bmc Plant Biol 16 doi:10.1186/s12870-016-0771-y

Walford S, Wu Y, Llewellyn DJ, Dennis ES (2011) GhMYB25-like: a key factor in early cotton fibre development The Plant Journal 65:785-797 doi:10.1111/j.1365-313X.2010.04464.x

Wang $\mathrm{F}$ et al. (2015) Identification and Characterization of 40 Isolated Rehmannia glutinosa MYB Family Genes and Their Expression Profiles in Response to Shading and Continuous Cropping Int J Mol Sci 16:15009-15030 doi:10.3390/ijms160715009

Wang X et al. (2019a) PatJAZ6 Acts as a Repressor Regulating JA-Induced Biosynthesis of Patchouli Alcohol in Pogostemon Cablin Int J Mol Sci 20:6038 doi:10.3390/ijms20236038Yan W, Yang Y, Wu Y, Yu J, Zhang J, Yang D, Muhammad ZUH (2021) Isopentenyl Diphosphate Isomerase (IPI) Gene 
615 Silencing Negatively Affects Patchouli Alcohol Biosynthesis in Pogostemon cablin Plant Mol Biol Rep

616 doi:10.1007/s11105-020-01269-0

617 Yusenko MV et al. (2020) Monensin, a novel potent MYB inhibitor, suppresses proliferation of acute

618 myeloid leukemia and adenoid cystic carcinoma cells Cancer Lett 479:61-70

619 doi:https://doi.org/10.1016/j.canlet.2020.01.039

620 Zhang Z et al. (2017) Natural variation in CTB4a enhances rice adaptation to cold habitats Nat Commun

6218 doi:10.1038/ncomms14788

622

623 


\section{Supplementary Files}

This is a list of supplementary files associated with this preprint. Click to download.

- supplement.pdf 